\title{
Ganancia de peso en ovinos de pelo tipo Sudán: Efecto de la granja, sexo y tipo de parto ${ }^{1}$
}

\author{
Clara V. Rúa-Bustamante ${ }^{2}$, Maximiliano Ambrosio ${ }^{3}$ \\ y Abner A. Rodríguez-Carías ${ }^{4}$
}

J. Agric. Univ. P.R. 103(2):173-182 (2019)

\begin{abstract}
RESUMEN
Se evaluó la ganancia diaria de peso (GDP) de ovinos de pelo tipo Sudán en crecimiento utilizando 1,090 registros tomados durante dos años en tres granjas localizadas en el trópico seco en la región del Caribe de Colombia. En cada una de las granjas se registró el tipo de parto de la oveja y el sexo de los corderos. Para determinar la GDP, los corderos se pesaron semanalmente desde el nacimiento hasta los 240 días de edad. Los datos se analizaron según un diseño estadístico completamente aleatorizado con arreglo factorial 3 (granjas) x 2 (sexo del cordero) x 3 (tipo de parto, sencillo, doble o triple). Se utilizó la prueba de Tukey para la separación de medias. La GDP fue similar entre las tres granjas evaluadas con un promedio general de $112 \mathrm{~g}$. Se observó en promedio una mayor $(P<0.05)$ GDP en hembras que en machos ( 115 versus $103 \mathrm{~g})$ y tal como esperado, la GDP individual de corderos producto de partos sencillos $(117 \mathrm{~g})$ fue mayor $(\mathrm{P}<0.05)$ que la de corderos nacidos de partos dobles (106 g) o triples $(23 \mathrm{~g})$. La mayor GDP de corderas se observó en dos de las tres granjas. La GDP fue similar en machos o hembras nacidos de partos sencillos, sin embargo, en corderas nacidas de partos dobles la GDP fue mayor $(P<0.05)$ que en corderos, y lo contrario se observó en partos triples donde la GDP fue $32 \mathrm{~g}$ mayor $(P<0.05)$ en machos que en hembras. En resumen, la GDP de ovinos de pelo tipo Sudán en la región del Caribe de Colombia es buena dado su sistema de crianza extensivo. La GDP fue similar entre fincas pero difirió entre el sexo de la cría y el tipo de parto.
\end{abstract}

Palabras clave: ovino de pelo, crecimiento, trópico seco

\section{ABSTRACT}

Weight gain in Sudan hair sheep: Effect of farm, sex and type of parturition

The daily weight gain (DWG) of growing Sudan-type hair sheep was evaluated using 1,090 records taken over two years at three farms located in the dry tropics of the Colombian Caribbean region. On each farm, the type of

${ }^{1}$ Manuscrito sometido a la Junta Editorial el 10 de octubre de 2018.

${ }^{2}$ Investigador, Corporación Colombiana de Investigación Agropecuaria. e-mail: crua@ agrosavia.co

${ }^{3}$ Especialista Técnico, Asociación de Criadores de Ganado Ovino de Colombia.

${ }^{4}$ Catedrático, Departamento de Ciencia Animal, Universidad de Puerto Rico, Mayagüez. e-mail: abner.rodriguez3@upr.edu 
birth (single or multiple) and the sex of lambs were recorded. To determine DWG, 1,090 lambs were weighed weekly from birth to $\mathbf{2 4 0}$ days of age. The data were analyzed according to a completely randomized statistical design with factorial arrangement 3 (farms) $\times 2$ (sex of the lamb) $\times 3$ (type of delivery, single, double or triple). The Tukey test for the separation of means was used. The DWG was similar among the three farms averaging $112 \mathrm{~g}$. A higher $(P<0.05)$ DWG was observed in females than in males (115 versus $103 \mathrm{~g}$ ), and as expected, the individual DWG of lambs from single calving (117 g) was higher $(P<0.05)$ than twin $(106 \mathrm{~g})$ or triple $(23 \mathrm{~g})$. The highest DWG of ewe lambs than in males was observed on two of the three farms. The DWG was similar in males and females born from single births; however, in ewe lambs born from double births, DWG was higher $(P<0.05)$ than in males, and the opposite was observed in triple deliveries where DWG was $32 \mathrm{~g}$ higher $(P<0.05)$ in males than in females. In summary, DWG of Sudan hair sheep in the Caribbean region of Colombia is good given its extensive sheep raising system. The DWG was similar among farms but differed between the sex of the lamb and the type of parturition.

Key words: hair sheep, growth, dry tropics

\section{INTRODUCCIÓN}

En Colombia, el ovino de pelo criollo es también conocido con el nombre de camuro, africana, o pelona, según las regiones donde se críe. En este tipo de ovino, se han reportado altos niveles de variabilidad genética debido principalmente a las diversas rutas de ingreso al continente americano, probablemente durante el descubrimiento y la conquista. Similar a los bovinos, el banco genético de ovinos de pelo incluye animales provenientes de la península Ibérica, otros países de Europa y las islas Canarias (Vivas-Ascue, 2013). El ovino de pelo se caracteriza por su alta fertilidad y capacidad prolífica, su baja presencia de enfermedades y su virtud de utilizar fuentes alimenticias alternas como subproductos de cosecha (Arcos et al., 2002). Se ha reportado la presencia de ovinos de pelo tipo Sudán, Etíope y Abisinio en el continente americano, mayormente en la región del Caribe (Vivas-Ascue, 2013). Los tres tipos poseen similar número de alelos, lo que indica la alta diversidad genética (Vivas-Ascue, 2013). En Colombia, están presentes los tipos Etiope y Sudán, de adaptación al medio ambiente seco tropical y que se caracterizan por su rusticidad, mansedumbre, prolificidad y excelente comportamiento. Estas características convierten a estos ovinos en una innegable alternativa para contribuir al desarrollo de las comunidades, especialmente las menos favorecidas que son las que más conocen y mantienen la especie.

Actualmente para la producción de ovinos, predominan sistemas de producción de trashumancia, nómadas, estancias, sistemas extensivos en zonas marginales, sistemas intensivos y sistemas mixtos (Herrera et al., 2010). Estos sistemas se catalogan como aquellos con media 
incorporación de tecnología y son considerados de economía familiar, con integraciones de actividades agrícolas y pecuarias como fuente de ingresos y que poseen un mediano nivel de adopción de tecnología, que solo les permiten incorporar algunos aspectos de manejo zootécnico, sanitario y administrativo (Castellanos et al., 2010).

En la región del Caribe de Colombia, los ovinos de pelo se crían principalmente en sistemas de producción extensivos o mixtos combinados con la ganadería bovina. Sin embargo, es poca la información documentada sobre el comportamiento productivo de ovinos de pelo tipo Sudán criado bajo estas condiciones. Es además bien conocido que el entorno o granja, el sexo del animal y el tipo o cantidad de crías por parto son factores extrínsecos o intrínsecos que influyen sobre el crecimiento de los corderos durante los períodos de lactancia y engorde (De Lucas et al., 2003). Diversos estudios han demostrado que corderos provenientes de partos simples presentan una mayor tasa de crecimiento pre y postdestete con respecto a aquellos provenientes de partos gemelares y que los machos presentan un mayor peso al nacimiento así como una mayor ganancia de peso pre y post-destete que las hembras (González-Garduño et al., 2002). Otros trabajos, sin embargo, realizados con distintas razas y bajo diferentes sistemas de producción no indican diferencias atribuidas al sexo para el peso al nacimiento de los corderos (Quintero et al., 1997) ni para el crecimiento pre-destete (González-Garduño et al., 2010). Inclusive algunos autores encontraron mayores tasas de crecimiento a los 30 y 60 días para las hembras, no existiendo diferencia en el peso al destete para los corderos de ambos sexos (Gbangboche et al., 2006).

Dada la importancia del ovino de pelo, debido a su mayor resistencia al estrés por calor, para el desarrollo del sector ovino en Colombia, el objetivo de esta investigación fue determinar el efecto de la granja, el sexo del animal y el tipo de parto de la oveja sobre la ganancia diaria de peso de corderos de pelo tipo Sudán.

\section{MATERIALES Y MÉTODOS}

Se utilizaron 1,090 datos recolectados en tres granjas con características similares de sistema de producción durante los años 2013 y 2014. Las granjas evaluadas se encuentran ubicadas en el corregimiento de Mariangola a $10^{\circ} 10^{\prime} 53.3^{\prime \prime}$ de latitud norte y $73^{\circ} 35^{\prime} 45.6$ " de longitud oeste, en el municipio de Valledupar en la microrregión Valle del César del departamento del César, República de Colombia, región Caribe. La zona presenta una temperatura promedio anual de $28.4^{\circ}$ $\mathrm{C}$, humedad relativa que oscila entre 56 y $74 \%$, precipitación anual promedio de $970 \mathrm{~mm}$, con distribución bimodal de los meses de mayo 
a junio y de agosto a noviembre y altura sobre el nivel del mar de 100 metros. Se considera una microrregión de sucesión ecológica: bosque seco tropical, sabanas (Grajales et al., 2016).

En la granja A, el área total es de 950 hectáreas dedicadas a la ganadería bovina (carne y leche) y ganadería ovino-caprina (carne). El sistema de alimentación para los ovinos es pastoreo extensivo mixto (bovinos, ovinos y caprinos), los ovinos y caprinos pastorean de manera independiente en las áreas dedicadas a la producción bovina bajo un manejo rotacional diseñado según la temporada seca y lluviosa. Las praderas están cubiertas de gramíneas introducidas (Panicum maximum cv. Tanzania), gramíneas arvenses, arbóreas y arbustivas nativas. En la ganadería ovina el fenotipo racial es el ovino criollo de pelo Sudán.

En la granja B, el área total es de 550 hectáreas dedicadas a la ganadería bovina de hembras y machos (carne) y ganadería ovino-caprina (carne). El sistema de alimentación para los ovinos es también el pastoreo extensivo mixto (bovinos, ovinos y caprinos), los ovinos y caprinos pastorean de manera independiente en las áreas dedicadas a la producción bovina bajo un manejo rotacional de acuerdo a la temporada seca y lluviosa. Las praderas están cubiertas de gramínea nativa (Bothriochloa pertusa), arvenses, arbóreas y arbustivas nativas, observándose baja oferta forrajera aparentemente por la calidad de sus suelos y menor incidencia de precipitaciones. En la ganadería ovina el fenotipo racial es el ovino criollo de pelo Sudán en un $21 \%$, ovinos de la raza Blackbelly en un $33 \%$ y cruzamientos de hembras criollas de pelo Sudán con machos de la raza Dorper y BlackBelly en un $46 \%$.

En la granja C, el área total es de 550 hectáreas dedicadas al cultivo de palma de aceite bajo sistema de riego por aspersión, ganadería bovina de engorde (carne) y ganadería ovina (carne). El ganado ovino pastorea los corredores del cultivo de palma para el control de arvenses, alcanzando a consumir gramíneas nativas (pastoreo conducido); y se alterna pastoreando con los bovinos (pastoreo mixto) en praderas cubiertas de gramínea introducida (Panicum maximum cv. Tanzania), gramíneas, arvenses, arbóreas y arbustivas nativas bajo manejo rotacional. En la ganadería ovina el fenotipo racial es el ovino criollo de pelo Sudán en un $38 \%$ y cruzamientos de hembras criollas de pelo Sudán con machos de la raza Dorper y Kathadin en un $62 \%$.

En las tres granjas evaluadas los corderos son retenidos en el corral de manera permanente durante los primeros 10 a 15 días de nacidos, permitiendo que la oveja salga a pastoreo durante el día y regrese en el momento que desee. El manejo reproductivo se realiza en temporadas de montas y de partos (cinco grupos de animales divididos en cinco temporadas de monta durante el año), adaptado del sistema STAR (Lewis 
et al., 1996) sin la clasificación por lotes. La proporción machos:hembra es de 1:33 a 1:35 y el producto de venta es el cordero (hembras y machos) de 30 a 35 kilogramos de peso, con una edad entre ocho y diez meses. En los machos se realiza castración con elastrador entre los tres a siete días de vida. De igual manera en las tres granjas los ovinos son suplementados con sal mineralizada formulada según los minerales presentes en el suelo y con adición de coccidiostato, vitaminas, arcillas y jabones cálcicos, en una dosis de 10 gramos por animal por día.

En cada finca, en ambos años, se documentó la fecha de parto de cada oveja y se registró la información de los factores sexo y tipo de parto. Para la variable de ganancia de peso diario, se realizaron pesajes mensuales de los machos y hembras desde el nacimiento hasta los 240 días de edad (edad promedio en la que se alcanza el peso al sacrificio) utilizando una báscula de reloj.

Los datos de ganancia en peso se analizaron según un diseño completamente aleatorizado con arreglo factorial de tratamientos 3 (granja) x 2 (sexo del cordero) x 3 (tipo de parto) utilizando el paquete estadístico de SAS (2009). Se utilizó la prueba de Tukey para la separación de medias.

\section{RESULTADOS Y DISCUSIÓN}

Los valores promedio de ganancia diaria de peso (GDP) de los efectos principales se presentan en el Cuadro 1. La GDP fue similar entre las tres granjas evaluadas (117, 105 y $114 \mathrm{~g} / \mathrm{d}$ para la granja A, B y C, respectivamente), lo que se explica por la similitud en animales, en prácticas de manejo y en condiciones climáticas entre localidades. En las tres granjas en conjunto, la GDP tuvo un promedio de $112 \mathrm{~g}$, valor que se considera excelente para el crecimiento de corderos criados bajo

CuAdRo 1.-Efecto de la granja, el sexo del cordero y el tipo de parto sobre la ganancia diaria de peso (GDP) en ovinos de pelo con genotipo tipo Sudán.

\begin{tabular}{llccc}
\hline Componente & & $\mathrm{N}$ & $\mathrm{GDP}$ & $\mathrm{P}$ \\
\hline Granja & $\mathrm{A}$ & 179 & $117 \pm 0.46$ & NS \\
& $\mathrm{B}$ & 340 & $105 \pm 0.46$ & \\
\multirow{3}{*}{ Sexo } & $\mathrm{C}$ & 571 & $114 \pm 0.54$ & \\
& Hembra & $812 \mathrm{a}^{1}$ & $115 \pm 0.51$ & 0.01 \\
Tipo parto & Macho & $278 \mathrm{~b}$ & $103 \pm 0.49$ & \\
& Sencillo & $599 \mathrm{a}$ & $117 \pm 0.51$ & 0.01 \\
& Doble & $468 \mathrm{~b}$ & $106 \pm 0.50$ & \\
& Triple & $23 \mathrm{c}$ & $99 \pm 0.45$ & \\
\hline
\end{tabular}

${ }^{1}$ Medias con diferente letra en el mismo componente difieren $\mathrm{P}<0.05$ 
sistemas extensivos de producción y coincide con GDP de 80 a 160 g reportados para Colombia por la Organización de las Naciones Unidas para la Alimentación y la Agricultura (FAO, por sus siglas en inglés) relacionados con nivel tecnológico medio (Garay y Correa, 2013). Se observó también una mayor $(\mathrm{P}<0.05)$ GDP en hembras que en machos (115 vs. 103 g, respectivamente), resultado que coincide o difiere de estudios anteriores que evaluaron la ganancia en peso en corderos bajo condiciones extensivas. Tal como esperado, la GDP individual de corderos producto de partos sencillos $(117 \mathrm{~g})$ fue mayor $(\mathrm{P}<0.05)$ que la de corderos nacidos de partos dobles (106 g) o triples (23 g).

Otros estudios efectuados en ovinos criados en sistemas de producción tanto extensiva como intensiva con o sin suplementación proteica, energética o mineral reportaron valores de GDP similares a los de este experimento. En Venezuela se realizó un estudio con corderos pre-destete de la raza Africana Oeste criados en condiciones agroecológicas clasificadas como bosque tropical muy seco (Dickson et al., 2004). Los ovinos en el citado estudio se criaron bajo condiciones de pastoreo y fueron suplementados con una ración comercial de alimento concentrado con el $17 \%$ de proteína cruda a razón de $300 \mathrm{~g} / \mathrm{d}$ y obtuvieron una GDP de $101 \mathrm{~g} /$ día, valor por debajo de los observados en este estudio. En ovinos de pelo raza Pelibuey de cuatro a ocho meses y de ocho a 12 meses de edad y alimentados en sistemas semi-intensivos con dietas con una proporción de forraje a concentrado de 40:60, se reportaron GDP de 104 y 112 g/d, respectivamente (Fonseca, 2003). En otro estudio, también con ovinos Pelibuey pero criados bajo condiciones intensivas, se observó efecto de sexo y el tipo de parto sobre la GDP de corderos (Macedo y Arredondo, 2008). En el citado estudio la GDP de corderos de partos sencillos, dobles y triples fue de 117, 106 y 99 gramos, respectivamente, resultados similares a los reportados en este estudio. Rastogi (2001) también reportó mayores GDP en corderos de la raza Black Belly nacidos de partos sencillos versus la de aquellos producto de partos dobles y triples.

El peso al nacimiento de animales de partos sencillos es mayor que en crías de partos múltiples (dobles o triples) ya que durante la gestación no tiene ninguna competencia por nutrientes y espacio en el útero de su madre (Macedo y Arredondo, 2008). Sin embargo, el peso total en conjunto de las crías favorece aquellos animales producto de partos múltiples.

En otra investigación (González-Garduño et al., 2002), los promedios de GDP de la etapa predestete y posdestete en corderos Blackbelly se encuentran dentro de los valores que se reportan en este estudio para las tres granjas. En ese estudio anterior se reportaron GDP de 88 g para hembras, $100 \mathrm{~g}$ para machos, $97 \mathrm{~g}$ para partos sencillos y $93 \mathrm{~g}$ para partos dobles. 
En el presente estudio, la GDP en las hembras fue mayor $(\mathrm{P}<0.05)$ que en los machos en dos de las tres fincas evaluadas (Figura 1). Estos resultados difieren de los reportados en ovinos Pelibuey criados en el clima cálido húmedo mexicano, donde las ganancias pre-destete en machos fue mayor que en hembras (104 vs. 90 g) (González-Garduño et al., 2010). En otro estudio con corderos de la raza Pelibuey (Hinojosa-Cuellar et al., 2012) también se observó que el peso al nacimiento y al destete y la GDP pueden estar influenciados por el sexo de la cría. En el último estudio la GDP fue mayor en hembras que en machos (200 vs. 100 g, respectivamente). En ovinos tipo West África, Persa Cabeza Negra, Blackbelly y Bergamasca, en pastoreo y suplementados eventualmente con pasto de corte, caña de azúcar y la leguminosa Leucaena leucochephala, se observó GDP postdestete de $104.8 \mathrm{~g}$ para machos y de $93.9 \mathrm{~g}$ para hembras.

La Figura 2 muestra los valores de GDP de la interacción sexo y tipo de parto. La GDP fue similar en animales nacidos de partos sencillos. En hembras nacidas de partos dobles la GDP fue mayor $(\mathrm{P}<0.05)$ que en machos, sin embargo, lo contrario se observó en partos triples, donde la GDP fue $32 \mathrm{~g}$ mayor $(\mathrm{P}<0.05)$ en machos que en hembras. Este tipo de comportamiento en GDP entre sexos como respuesta al parto podría estar relacionado a muchos factores no evaluados en este experimento, como el índice de condición corporal de las ovejas, su ali-

180

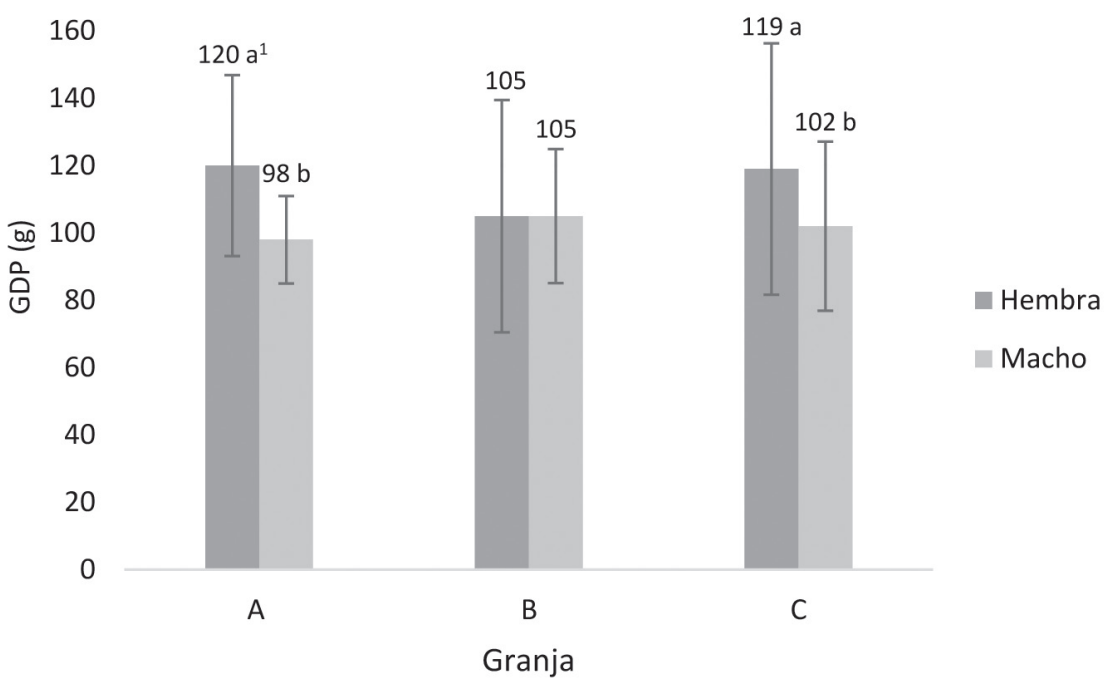

${ }^{1}$ Medias con diferente letra en la misma granja difieren $\mathrm{P}<0.05$ Sudán.

FiguRA 1. Interacción entre la granja y el sexo sobre la GDP de ovinos de pelo tipo 


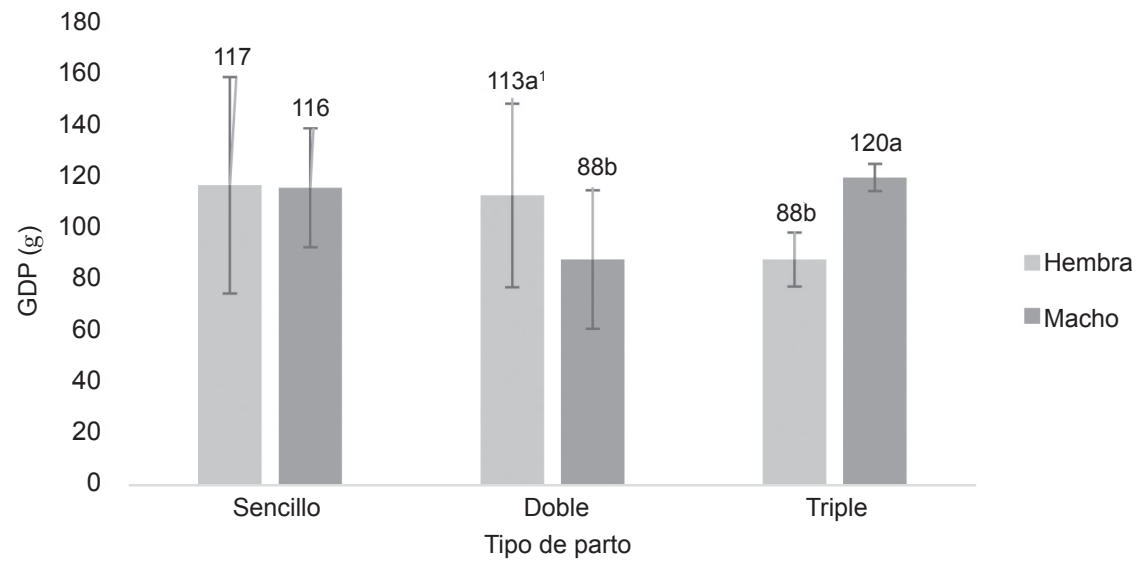

${ }^{1}$ Medias con diferente letra en el mismo tipo de parto difieren $\mathrm{P}<0.05$

FiguRA 2. Interacción entre el tipo de parto y el sexo sobre la GDP de ovinos de pelo tipo Sudán.

mentación durante el último tercio de la gestación, su capacidad de producción de calostro y leche y su instinto materno. Estos factores han sido asociados al peso al nacer de corderos nacidos de ovejas criadas en sistemas de producción extensivos (Martin et al., 2004).

En la Figura 3 se puede observar la interacción entre granja y el tipo de parto sobre la GDP de corderos tipo Sudán. Excepto en la granja

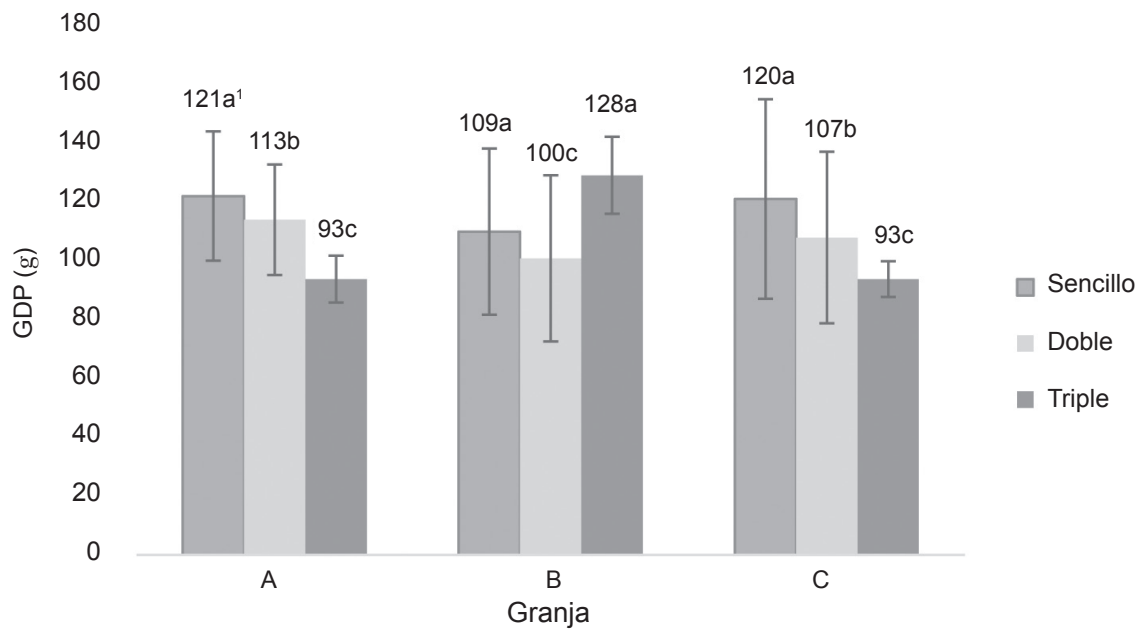

${ }^{1}$ Medias con diferente letra en la misma granja difieren $\mathrm{P}<0.05$

FIGURA 3. Interacción entre el tipo de parto y la granja sobre la GDP de ovinos de pelo tipo Sudán. 
B, y tal como esperado, la GDP de los corderos nacidos de partos sencillos fue mayor $(\mathrm{P}<0.05)$ que en aquellos nacidos de partos gemelares 0 triples. La diferencia en ganancia en peso entre partos sencillos y múltiples fue de 8 (doble) y 28 (triple) g/d en la granja A y de 13 (doble) y 27 (triple) g/d en la granja C. Sin embargo, un comportamiento atípico se observó en la granja B, donde la GDP de animales nacidos de partos triples fue $18 \mathrm{~g}$ mayor $(\mathrm{P}<0.05)$ que en aquellos de partos dobles y 19 $\mathrm{g}$ mayor que en animales nacidos de partos sencillos. En estudios relacionados, Carrillo y Segura (1993) reportaron GDP de 85 gramos para partos sencillos, 74 gramos para dobles y 72 gramos para partos múltiples en ovinos de raza Blackbelly y Pelibuey en México. En general, en este experimento los valores medios de GDP encontrados corresponden a los observados por otros autores en ovinos de pelo, donde los ovinos hembras y machos producto de nacimientos múltiples tienen una GDP individual menor que la de los nacidos de partos sencillos.

\section{CONCLUSIONES}

Dado el sistema de crianza y las condiciones climáticas prevalecientes, la GDP de ovinos de pelo tipo Sudán en la región del Caribe de Colombia puede considerarse como buena ( $>75 \mathrm{~g}$ ). Se observó consistencia en los resultados obtenidos entre las fincas evaluadas y se observó efecto del sexo de la cría y el tipo de parto sobre la GDP. Los resultados demuestran que este tipo de ovino representa una alternativa para sistemas de producción extensivos en regiones tropicales.

\section{LITERATURA CITADA}

Arcos, J.C., H. Romero, M.A. Vanegas y E. Riveros, 2002. Ovinos colombianos de pelo: Alternativa productiva para el sur del departamento del Tolima. Bucaramanga, Colombia: Corporación Colombiana de Investigación Agropecuaria (CORPOICA).

Carrillo, L. y J.C. Segura, 1996. Environmental and genetic effects on preweaning growth performance of hair sheep in Mexico. Trop Anim Health Prod. 25(3):173-178.

Castellanos, J.G., J.C. Rodríguez, W.L. Toro y C.L. Luengas, 2010. Agenda prospectiva de investigación y desarrollo tecnológico para la cadena productiva cárnica ovino-caprina en Colombia. Bogotá, Colombia: Ministerio de Agricultura y Desarrollo Rural.

De Lucas, T.J., L.A. Zarco, E. González, J. Tórtora, A. Villa-Godoy y C. Vázquez, 2003. Crecimiento predestete de corderos en sistemas intensivos de pastoreo y manejo reproductivo en el altiplano central de México. Vet Mex. 34(3): 235-245.

Dickson-Urdaneta L., G. Torres-Hernández, R. Dáubeterre y O. García, 2004. Crecimiento en ovinos West African bajo un sistema de pastoreo restringido en Venezuela. Rev Fac Agron (LUZ). 21: 59-67.

Fonseca, N., 2003. Contribución al estudio de la alimentación del ovino Pelibuey en Cuba. 2003 Dissertation Doctoral. La Habana, Cuba: Instituto de Ciencia Animal.

Garay, A.A. y G. Correa, 2013. Tecnología en la ovinocultura colombiana: estado del arte. Rev. Cien. Anim. 6(1): 125-142.

Gbangboche, A.B., M. Adamou-Ndiaye, A.K.I. Youssao, F. Farnir, J. Detilleux, F.A. Abiola y P.L. Leroy, 2006. Non-genetic factors affecting the reproduction performance, 
lamb growth and productivity indices of Djallonke sheep. Small Ruminant Res. 64(1-2): 133-142.

González-Garduño, R., G. Torres-Hernández y M. Castillo-Álvarez, 2002. Crecimiento de corderos Blackbelly entre el nacimiento y el peso final en el trópico húmedo de México. Vet Mex. 33(4): 443-453.

González-Garduño, R., G. Torres-Hernández y J. Arece-García, 2010. Comportamiento productivo y reproductivo de ovinos Pelibuey en un sistema de pariciones aceleradas con tres épocas de empadre al año. Zoot Trop. 28(1): 51-56.

Grajales-Lombana, H.A., G.A. Téllez y O.F. Ospina, 2016. Plan Estratégico Regional de Ciencia, Tecnología e Innovación (PERCTI) Departamento del Cesar. http://www. colciencias.gov.co/sites/default/files/upload/paginas/pedcti-cesar.pdf.

Herrera, J., H. Jordan y A.F. Senra, 2010. Aspectos del manejo y alimentación de la reproductora ovina Pelibuey en Cuba. Rev. Cub. Cienc. Agric. 44(3): 211-219.

Hinojosa-Cuéllar, J.A., J. Oliva-Hernández, G. Torres-Hernández, J.C.Segura-Correa, E.M. Aranda-lbáñez y J.M. González-Camacho, 2012. Factores que afectan el crecimiento predestete de corderos Pelibuey en el trópico húmedo de México. Universidad y Ciencia 28(2): 163-171.

Lewis, R.M., D.R. Notter, D.E. Hogue y B.H. Magee, 1996. Ewe fertility in the STAR accelerated lambing system. J. Anim. Sci. 74(7): 1511-1522.

Macedo, R. y V. Arredondo, 2008. Efecto del sexo, tipo de nacimiento y lactancia sobre el crecimiento de ovinos Pelibuey en manejo intensivo. Arch. Zootec. 57(218): 219-228.

Martin, G.B., J.T. Milton, H.D. Davidson, G.E. Banchero, D. Hunzicker, R. Lindsay y D. Blache, 2004. Natural methods for increasing reproductive efficiency in small ruminants. Animal Reproduction Sci. 82-83: 231-245.

Quintero, A., J. Boscan, R. Palomares, A. Gonzalez y J. Boissiere. 1997. Efecto del sexo sobre el peso corporal a diferentes edades en corderos West-African criados en el trópico venezolano. Arch. Latinoam. Prod. Anim. 5(1): 426-427.

Rastogi, R.K., 2001. Production performance of Barbados blackbelly sheep in Tobago, West Indies. Small Ruminant Res. 41(2): 171-175.

SAS Institute Inc., 2009. SAS/STAT® 9.2 software. Cary, NC: SAS Institute Inc.

Vivas-Ascue, N.J., 2013. Diversidad genética de ovinos criollos colombianos. Disertación Doctoral. Bogotá, Colombia: Universidad Nacional de Colombia. 